Industrial Engineering

\& Management Systems

Vol 12, No 1, March 2013, pp.2-8

http://dx.doi.org/10.7232/iems.2013.12.1.002

ISSN 1598-7248 | EISSN 2234-6473 |

(C) 2013 KIIE

\title{
Stability Analysis of Linear Uncertain Differential Equations
}

\author{
Xiaowei Chen* \\ Department of Risk Management and Insurance, Nankai University, Tianjin, China \\ Jinwu Gao \\ School of Information, Renmin University of China, Beijing, China
}

(Received: August 12, 2012 / Revised: December 7, 2012 / Accepted: March 6, 2013)

\begin{abstract}
Uncertainty theory is a branch of mathematics based on normolity, duality, subadditivity and product axioms. Uncertain process is a sequence of uncertain variables indexed by time. Canonical Liu process is an uncertain process with stationary and independent increments. And the increments follow normal uncertainty distributions. Uncertain differential equation is a type of differential equation driven by the canonical Liu process. Stability analysis on uncertain differential equation is to investigate the qualitative properties, which is significant both in theory and application for uncertain differential equations. This paper aims to study stability properties of linear uncertain differential equations. First, the stability concepts are introduced. And then, several sufficient and necessary conditions of stability for linear uncertain differential equations are proposed. Besides, some examples are discussed.
\end{abstract}

Keywords: Uncertain Process, Uncertain Differential Equation, Stability Analysis

* Corresponding Author, E-mail: jgao@ruc.edu.cn

\section{INTRODUCTION}

Differential equation is a classic mathematical branch widely applied in physics, engineering, biology, economics and other fields. Due to the influence of randomness, stochastic differential equation, a type of differential equation driven by Brownian motion, was founded by Ito (1951). Besides the Ito stochastic differential equations, Kunita and Watanabe (1967) introduced stochastic differential equations driven by square integrable martingales, and Meyer (1970) studied stochastic differential equations driven by square integrable semimartingales. After a half century development, the stochastic differential equation has been widely applied to physics, mechanics, biology, economics and finance, control theory, aerospace engineering, and other disciplines. It has become a basic modern mathematical tool to analyze the stochastic model for inspection system.

Note that the driven process Brownian motion is a stochastic process such that almost all sample paths are continuous but non-Lipschitz continuous functions, that is to say, all sample paths have infinite length. Thus, the Brownian motion describes the irregular movement of pollen with infinite speed. In order to describe the irregular movement of pollen with finite speed, an uncertain counterpart of Brownian motion called canonical Liu process was introduced by Liu (2008). Canonical Liu process is a Lipschitz continuous uncertain process with stationary and independent increments, and each increment is a normal uncertain variable. Following the canonical Liu process, uncertain calculus was initialized by Liu (2009) to deal with differentiation and integration of functions of uncertain processes. Furthermore, uncertain differential equation, a type of differential equation driven by the canonical Liu process, was defined by Liu (2008). Recently, Chen and Liu (2010) proved the existence and uniqueness theorem of solution for uncertain differential equation under the Lipschitz condition and linear growth condition. Moreover, uncertain differential equations have been applied to uncertain op- 
timal control by Zhu (2010) and uncertain financial market by Chen (2011), Liu (2008) and Peng and Yao (2011). For exploring the recent developments of uncertainty theory, the readers may consult Liu (2010).

Stability analysis on differential equation is an important aspect in investigating the qualitative properties of differential equation by Lyapunov function approach. Some efforts have also been devoted to the application of the Lyapunov function method to design controls or to obtain sufficient conditions for the optimality of a given control. The stability analysis on stochastic differential equations was initialized by Kats and Krasovskii (1960) through the stochastic Lyapunov approach to the study of qualitative properties of stochastic differential equations. The theory of stochastic Lyapunov functions has been a successful tool for assessing its stability properties (Kushner, 1967; Khas'minskii, 1962,1980).

Since the canonical Liu process is more appropriate to describe the real existence noise, uncertain differential equations have advantages in describing dynamic systems. Stability analysis of uncertain differential equation also has a great practical and theoretical significance. The rest of the paper is organized as follows: preliminary concepts of uncertain processes are recalled in Section 2. The concepts of uncertain differential equation are recalled in Section 3. The definitions of stability for uncertain differential equation are introduced in Section 4. Several sufficient and necessary conditions of stability for linear uncertain differential equations are proved in Section 5. Finally, a brief summary is given in Section 6.

\section{PRELIMINARY}

Uncertain measure $M$ is a real-valued set-function on a $\sigma$-algebra $\mathrm{L}$ over a nonempty set $\Gamma$ satisfying normality, duality, subadditivity and product axioms. The triplet $(\Gamma, L, M)$ is called an uncertainty space.

Definition 1 (Liu, 2007). An uncertain variable is a function from an uncertainty space $(\Gamma, L, M)$ to the set of real numbers, i.e., for any Borel set $B$ of real numbers, the set

$$
\{\xi \in B\}=\{\gamma \in \Gamma \mid \xi(\gamma) \in B\}
$$

is an event.

The uncertainty distribution function $\Phi: \Re \rightarrow[0,1]$ of an uncertain variable $\xi$ is defined as $\Phi(x)=M\{\xi \leq$ $x$. The expected value of an uncertain variable is defined as follows.

Definition 2 (Liu, 2007). Let $\xi$ be an uncertain variable. Then the expected value of $\xi$ is defined by

$$
E[\xi]=\int_{0}^{+\infty} M\{\xi \geq r\} \mathrm{d} r-\int_{-\infty}^{0} M\{\xi \leq r\} \mathrm{d} r
$$

provided that at least one of the two integrals is finite.
Liu (2007) proved the Markov inequality for uncertain variables. Let $\xi$ be an uncertain variable. Then for any given number $t>0$ and $p>0$, we have

$$
M\{|\xi| \geq t\} \leq \frac{E\left[|\xi|^{p}\right]}{t^{p}} .
$$

An uncertain process is essentially a sequence of uncertain variables indexed by time or space. The mathematical definition was introduced by Liu (2009).

Definition 3 (Liu, 2008). Let $\mathrm{T}$ be an index set and let $(\Gamma, L, M)$ be an uncertainty space. An uncertain process is a measurable function from $T \times(\Gamma, L, M)$ to the set of real numbers, i.e., for each $t \in T$ and any Borel set $B$ of real numbers, the set

$$
\left\{X_{t} \in B\right\}=\left\{\gamma \in \Gamma \mid X_{t}(\gamma) \in B\right\}
$$

is an event.

An important uncertain process called the canonical Liu process, which could be seen as the uncertain version of Wiener process, is difined as follows.

Definition 4 (Liu, 2009). An uncertain process $C_{t}$ is said to be a canonical Liu process if

(i) $C_{0}=0$ and almost all sample paths are Lipschitz continuous,

(ii) $C_{t}$ has stationary and independent increments,

(iii) every increment $C_{t+s}-C_{s}$ is a normal uncertain variable with expected value 0 and variance $t^{2}$, whose uncertainty distribution is

$$
\Phi(x)=\left(1+\exp \left(-\frac{\pi x}{\sqrt{3} t}\right)\right)^{-1}, x \in \mathfrak{R} .
$$

Definition 5 (Liu, 2009). Let $X_{t}$ be an uncertain process and let $C_{t}$ be a canonical Liu process. For any partition of cloesd interval $[a, b]$ with $a=t_{1}<t_{2}<\cdots$ $<t_{m+1}=b$, the mesh is written as

$$
\Delta=\max _{1 \leq i \leq k}\left|t_{i+1}-t_{i}\right|
$$

Then, the uncertain integral of $X_{t}$ with respect to $C_{t}$ is

$$
\int_{a}^{b} X_{t} \mathrm{~d} C_{t}=\lim _{\Delta \rightarrow 0} \sum_{i=1}^{k} X_{t_{i}} \cdot\left(C_{t_{i+1}}-C_{t_{i}}\right)
$$

provided that the limit exists almost surely and is an uncertain variable.

Let $C_{t}$ be a canonical Liu process and let $f(t)$ be an integrable function with respected to $t$. It has been proved that the uncertain integral $\int_{0}^{s} f(t) \mathrm{d} C_{t}$ is a normal uncertain variable with expected value 0 and variance $\left(\int_{0}^{s}|f(t)| \mathrm{d} t\right)^{2}$. Let $h(t, c)$ be a continuously differenti- 
able function. Then, $X_{t}=h\left(t, C_{t}\right)$ is an uncertain process. Liu (2009) proved the following chain rule

$$
\mathrm{d} X_{t}=\frac{\partial h}{\partial t}\left(t, C_{t}\right) \mathrm{d} t+\frac{\partial h}{\partial c}\left(t, C_{t}\right) \mathrm{d} C_{t} .
$$

\section{UNCERTAIN DIFFERENTIAL EQUATIONS}

Based on the canonical Liu process, Liu (2008) introduced uncertain differential equation to describe dynamic uncertain systems.

Definition 6 (Liu, 2008). Suppose that $C_{t}$ is a canonical Liu process, and $f$ and $g$ are some given functions. Then,

$$
\mathrm{d} X_{t}=f\left(t, X_{t}\right) \mathrm{d} t+g\left(t, X_{t}\right) \mathrm{d} C_{t}
$$

is called an uncertain differential equation. A solution is an uncertain process $X_{t}$ that satisfies (2) identically in $t$.

The following uncertain differential equation

$$
\mathrm{d} X_{t}=a(t) X_{t} \mathrm{~d} t+b(t) X_{t} \mathrm{~d} C_{t}
$$

is called a homogeneous linear uncertain differential equation. It has a unique solution

$$
X_{t}=X_{0} \exp \left(\int_{0}^{t} a(s) \mathrm{d} s+\int_{0}^{t} b(s) \mathrm{d} C_{s}\right)
$$

Note that $X_{t}$ is a lognormal uncertain variable for each $t$. Suppose that $u_{1 t}, u_{2 t}, v_{1 t}, v_{2 t}$ are some continuous functions with respect to $t$. It has been proved by Chen and Liu (2010) that the linear uncertain differential equation

$$
\mathrm{d} X_{t}=\left(u_{1 t} X_{t}+u_{2 t}\right) \mathrm{d} t+\left(v_{1 t} X_{t}+v_{2 t}\right) \mathrm{d} C_{t}
$$

has a solution

$$
X_{t}=U_{t}\left(X_{0}+\int_{0}^{t} \frac{u_{2 s}}{U_{s}} \mathrm{~d} s+\int_{0}^{t} \frac{v_{2 s}}{U_{s}} \mathrm{~d} C_{s}\right)
$$

where

$$
U_{t}=\exp \left(\int_{0}^{t} u_{1 r} \mathrm{~d} r+\int_{0}^{t} v_{1 r} \mathrm{~d} C_{r}\right)
$$

Liu (2012) give two analytic methods to solve uncertain differential equations. We will introduced as follows.

Theorem 1 (Liu, 2011). Let $f$ be a function of two variables and let $\sigma_{t}$ be an integrable uncertain process. Then the uncertain differential equation

$$
\mathrm{d} X_{t}=f\left(t, X_{t}\right) \mathrm{d} t+t X_{t} \mathrm{~d} C_{t}
$$

has a solution $X_{t}=Y_{t}^{-1} Z_{t}$ where $Y_{t}=\exp \left(-\int_{0}^{t} \sigma_{s} \mathrm{~d} C_{s}\right)$ and
$Z_{t}$ is the solution of uncertain differential equation

$$
\mathrm{d} Z_{t}=Y_{t} f\left(t, Y_{t}^{-1} Z_{t}\right) \mathrm{d} t
$$

with initial value $Z_{0}=X_{0}$.

Theorem 2 (Liu, 2011). Let $g$ be a function of two variables and let $t$ be an integrable uncertain process. Then the uncertain differential equation

$$
\mathrm{d} X_{t}=t X_{t} \mathrm{~d} t+g\left(t, X_{t}\right) \mathrm{d} C_{t}
$$

has a solution $X_{t}=Y_{t}^{-1} Z_{t}$ where $Y_{t}=\exp \left(-\int_{0}^{t} \sigma_{s} \mathrm{~d} s\right)$ and $Z_{t}$ is the solution of uncertain differential equation $\mathrm{d} Z_{t}$ $=Y_{t} g\left(t, Y_{t}^{-1} Z_{t}\right) \mathrm{d} C_{t}$ with initial value $Z_{0}=X_{0}$.

\section{CONCEPTS OF STABILITY}

Before we study the stability properties of uncertain differential equations, we will introduce some stability concepts.

Definition 7 (Liu, 2009). An uncertain differential equation is said to be stable if any solutions $X_{t}$ and $Y_{t}$ satisfies

$$
\lim _{\left|X_{0}-Y_{0}\right| \rightarrow 0} M\left|X_{t}-Y_{t}\right|>\varepsilon=0
$$

for any given number $\varepsilon>0$ and any time $t>0$.

In fact, an uncertain differential equation is stable if and only if for any given $\kappa$ and $\varepsilon$, there exists $\delta$ such

$$
M\left\{\left|X_{t}-Y_{t}\right|>\kappa\right\}<\varepsilon, t>0
$$

whenever $\left|X_{0}-Y_{0}\right|<\delta$. The uncertain differential equation

$$
\mathrm{d} X_{t}=a \mathrm{~d} t+\sigma \mathrm{d} C_{t}
$$

has the solutions

$$
X_{t}=X_{0}+a t+\sigma C_{t} \text { and } Y_{t}=Y_{0}+a t+\sigma C_{t}
$$

with the initial values $X_{0}$ and $Y_{0}$, respectively. Then for any given nonnegative real number $\varepsilon$ and any time $t>0$, such that

$$
M\left\{\left|Y_{t}-X_{t}\right| \geq \varepsilon\right\}=M\left\{\left|Y_{0}-X_{0}\right| \geq \varepsilon\right\}=0
$$

when ever $\left|Y_{0}-X_{0}\right|<\varepsilon$. Thus this uncertain differential equation is stable. Next, we will give another example which is not stable. The uncertain differential equation

$$
\mathrm{d} X_{t}=\left(m-\alpha X_{t}\right) \mathrm{d} t+\sigma \mathrm{d} C_{t}, \alpha<0
$$

has solutions 


$$
X_{t}=\frac{m}{\alpha}+\left(X_{0}-\frac{m}{\alpha}\right) \exp (-\alpha t)+\sigma \int_{0}^{t} \exp (\alpha(s-t)) \mathrm{d} C_{s}
$$

and

$$
Y_{t}=\frac{m}{\alpha}+\left(Y_{0}-\frac{m}{\alpha}\right) \exp (-\alpha t)+\sigma \int_{0}^{t} \exp (\alpha(s-t)) \mathrm{d} C_{s},
$$

with the initial values $X_{0}$ and $Y_{0}$, respectively. Then for any given nonnegative real number $\varepsilon$, such that

$$
M\left\{\left|Y_{t}-X_{t}\right| \geq \varepsilon\right\}=M\left\{\exp (-\alpha t)\left|Y_{0}-X_{0}\right| \geq \varepsilon\right\}=1
$$

provided that $t$ is sufficiently large.

Definition 8. An uncertain differential equation is said to be asymptotically stable if it is stable and

$$
\lim _{t \rightarrow+\infty}\left|X_{t}-Y_{t}\right|=0 \text {, a.s. }
$$

whenever $\left|X_{0}-Y_{0}\right|<\delta$.

The stability and asymptotical stability properties of uncertain differential equation can be depicted by Figure 1 .

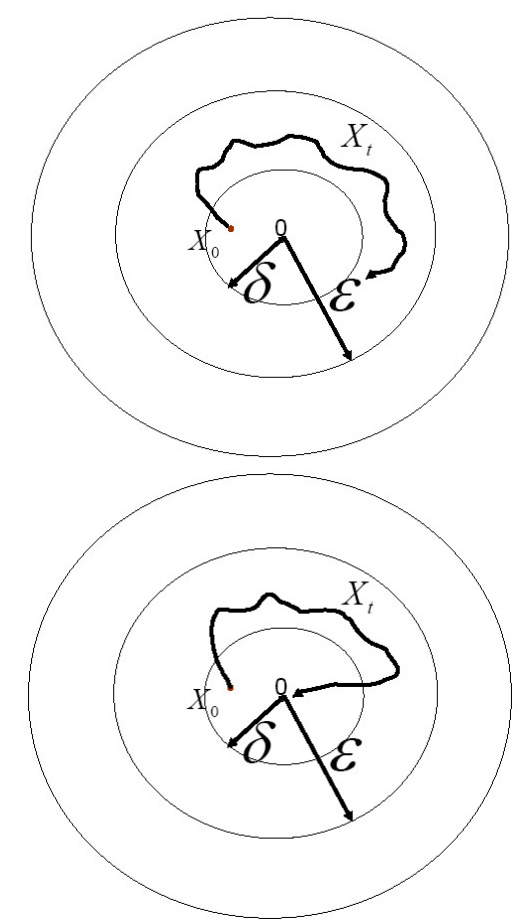

Figure 1. The stability and asymptotical stability of uncertain differential equation.

Definition 9. An uncertain differential equation is said to be global asymptotically stable if it is stable and

$$
\lim _{t \rightarrow+\infty}\left|X_{t}-Y_{t}\right|=0 \text {, a.s. }
$$

for any $X_{0}$ and $Y_{0}$.

\section{STABILITY THEOREMS}

Theorem 3. Suppose that $a(t)$ and $b(t)$ are continuous functions and $\int_{0}^{+\infty}|b(s)| \mathrm{d} s<+\infty$. Then the uncertain differential equation

$$
\mathrm{d} X_{t}=a(t) X_{t} \mathrm{~d} t+b(t) X_{t} \mathrm{~d} C_{t}
$$

is stable if and only if $\sup _{t \geq 0} \int_{0}^{t} a(s) \mathrm{d} s<+\infty$.

Proof. It has been proved that the unique solution of uncertain differential Eq. (6) is

$$
X_{t}=X_{0} \exp \left(\int_{0}^{t} a(s) \mathrm{d} s+\int_{0}^{t} b(s) \mathrm{d} C_{s}\right)
$$

which is a normal uncertain variable with expected value 0 and variance $\left(\int_{0}^{t}|b(s)| \mathrm{d} s\right)^{2}$ with respected to $t$. For any given positive number $\kappa$, we have

$$
\begin{aligned}
M\left\{\left|X_{t}-Y_{t}\right|>\kappa\right\} & =M\left\{\left|X_{0}-Y_{0}\right| \exp \left(\int_{0}^{t} a(s) \mathrm{d} s+\int_{0}^{t} b(s) \mathrm{d} C_{s}\right)>\kappa\right\} \\
& =M\left\{\int_{0}^{t} b(s) \mathrm{d} C_{s}>\ln \frac{\kappa}{\left|X_{0}-Y_{0}\right|}-\int_{0}^{t} a(s) \mathrm{d} s\right\} \\
& =1-\Phi_{t}\left(\ln \frac{\kappa}{\left|X_{0}-Y_{0}\right|}-\int_{0}^{t} a(s) \mathrm{d} s\right) .
\end{aligned}
$$

Then for any positive number $\varepsilon$, we get

$$
M\left\{\left|X_{t}-Y_{t}\right|>\kappa\right\}<\varepsilon \Leftrightarrow 1-\Phi_{t}\left(\ln \frac{\kappa}{\left|X_{0}-Y_{0}\right|}-\int_{0}^{t} a(s) \mathrm{d} s\right)<\varepsilon .
$$

On the one hand, we have

$$
\sup _{t \geq 0} \int_{0}^{t} a(s) \mathrm{d} s<+\infty \text { and } \int_{0}^{+\infty}|b(s)| \mathrm{d} s<+\infty .
$$

For any given positive numbers $\kappa$ and $\varepsilon$, there exists a number $\delta$, say

$$
\delta=\kappa \exp \left(-\frac{\sqrt{3}}{\pi} \int_{0}^{+\infty}|b(s)| \mathrm{d} s \ln \frac{1-\varepsilon}{\varepsilon}-\sup _{t \geq 0} \int_{0}^{t} a(s) \mathrm{d} s\right)
$$

such that

$$
\begin{aligned}
M\left\{\left|X_{t}-Y_{t}\right|>\kappa\right\} & =1-\Phi_{t}\left(\ln \frac{\kappa}{\left|X_{0}-Y_{0}\right|}-\int_{0}^{t} a(s) \mathrm{d} s\right) \\
& \leq 1-\Phi_{t}\left(\ln \frac{\kappa}{\delta}-\int_{0}^{t} a(s) \mathrm{d} s\right) \\
& \leq \varepsilon
\end{aligned}
$$

as $\left|X_{0}-Y_{0}\right|<\delta$. 
On the other hand, if Eq. (6) is stable, it follows from the definition of stability that for any given positive numbers $\kappa$ and $\varepsilon$ there exists a $\delta$ such that

$$
M\left\{\int_{0}^{t} b(s) \mathrm{d} C_{s}>\ln \frac{\kappa}{\left|X_{0}-Y_{0}\right|}-\int_{0}^{t} a(s) \mathrm{d} s\right\}<\varepsilon
$$

as $\left|X_{0}-Y_{0}\right|<\delta$. Since $\int_{0}^{t} b(s) \mathrm{d} C_{s}$ is a normal uncertain variable with expected value 0 and finite variance for all $t>0$. Thus

$$
\ln \frac{\kappa}{\left|X_{0}-Y_{0}\right|}-\int_{0}^{t} a(s) \mathrm{d} s \geq \Phi^{-1}(\varepsilon), \forall t>0 .
$$

Therefore we get

$$
\sup _{t \geq 0} \int_{0}^{t} a(s) \mathrm{d} s \leq \ln \frac{\kappa}{\delta}-\Phi^{-1}(\varepsilon)<+\infty .
$$

Thus the uncertain differential Eq. (6) is stable if and only if $\sup _{t \geq 0} \int_{0}^{t} a(s) \mathrm{d} s<+\infty$.

Example 1. Suppose that $a(t)$ and $b(t)$ are two functions

$$
a(t)=\frac{1}{1+t^{2}} \text { and } b(t)=\exp (-t)
$$

Then

$$
\sup _{t \geq 0} \int_{0}^{t} a(s) \mathrm{d} s=\frac{\pi}{2}<+\infty \text { and } \int_{0}^{+\infty}|b(s)| \mathrm{d} s=1<+\infty .
$$

Thus the uncertain differential equation

$$
\mathrm{d} X_{t}=\frac{X_{t}}{1+t^{2}} \mathrm{~d} t+\exp (-t) X_{t} \mathrm{~d} C_{t}
$$

is stable.

Theorem 4. Suppose that $a(s)$ and $b(s)$ are continuous functions and $\int_{0}^{+\infty}|b(s)| \mathrm{d} s<+\infty$. The uncertain differential equation

$$
\mathrm{d} X_{t}=a(t) X_{t} \mathrm{~d} t+b(t) X_{t} \mathrm{~d} C_{t}
$$

is asymptotically stable if and only if $\lim _{t \rightarrow+\infty} \int_{0}^{t} a(s) \mathrm{d} s=-\infty$.

Proof. It follows from Theorem 3 that the uncertain differential Eq. (8) is stable for

$$
\lim _{t \rightarrow+\infty} \int_{0}^{t} a(s) \mathrm{d} s=-\infty
$$

It has been proved that for any two solutions of Eq. (6) satisfying

$$
X_{t}-Y_{t}=\left(X_{0}-Y_{0}\right) \exp \left(\int_{0}^{t} a(s) \mathrm{d} s+\int_{0}^{t} b(s) \mathrm{d} C_{s}\right)
$$

Since $\int_{0}^{+\infty}|b(s)| \mathrm{d} s<+\infty$, we have $\int_{0}^{t} b(s) \mathrm{d} C_{s}<+\infty$, a.s. Therefore $\lim _{t \rightarrow \infty}\left|X_{t}-Y_{t}\right|=0$ if and only if

$$
\lim _{t \rightarrow+\infty} \int_{0}^{t} a(s) \mathrm{d} s=-\infty .
$$

Example 2. Suppose that $a(t)$ and $b(t)$ are two functions defined by

$$
a(t)=-1 \text { and } b(t)=\exp (-t) .
$$

Then

$$
\int_{0}^{+\infty} a(s) \mathrm{d} s=-\infty \text { and } \int_{0}^{+\infty}|b(s)| \mathrm{d} s=1<+\infty .
$$

Thus the uncertain differential equation

$$
\mathrm{d} X_{t}=-X_{t} \mathrm{~d} t+\exp (-t) X_{t} \mathrm{~d} C_{t}
$$

is asymptotically stable.

Theorem 5. Suppose that $a(t)$ and $b(t)$ are continuous functions with respect to $t$ and $\int_{0}^{+\infty}|b(s)| \mathrm{d} s=+\infty$. The uncertain differential Eq. (6) is asymptotically stable if there exists a number $p>1$ such that

$$
\lim _{T \rightarrow+\infty} \sup _{t \geq T} \frac{\int_{0}^{t} a(s) \mathrm{d} s}{\left(\int_{0}^{t}|b(s)| \mathrm{d} s\right)^{p}}<0
$$

Proof. For any given $\kappa$, we have

$$
\begin{gathered}
M\left\{\left|X_{t}-Y_{t}\right|>\kappa\right\}=M\left\{\left|X_{0}-Y_{0}\right| \exp \left(\int_{0}^{t} a(s) \mathrm{d} s+\int_{0}^{t} b(s) \mathrm{d} C_{s}\right)>\kappa\right\} \\
=M\left\{\frac{\int_{0}^{t} b(s) \mathrm{d} C_{s}}{\left(\int_{0}^{t}|b(s)| \mathrm{d} s\right)^{p}}>\frac{\ln \frac{\kappa}{\left|X_{0}-Y_{0}\right|}}{\left(\int_{0}^{t}|b(s)| \mathrm{d} s\right)^{p}}-\frac{\int_{0}^{t} a(s) \mathrm{d} s}{\left(\int_{0}^{t}|b(s)| \mathrm{d} s\right)^{p}}\right\} \\
=\frac{\left(\int_{0}^{t}|b(s)| \mathrm{d} s\right)^{2 p}\left(\frac{\ln \frac{\kappa}{\left|X_{0}-Y_{0}\right|}}{\left(\int_{0}^{t} \mid b(s) \mathrm{d} C_{s}\right)^{2}}-\frac{\left.\int_{0}^{t} a(s) \mathrm{d} s\right)^{p}}{\left.\left(\int_{0}^{t}|b(s)| \mathrm{d} s\right)^{p}\right)^{2}}\right.}{\left(\int_{0}^{t}|b(s)| \mathrm{d} s\right)^{2(p-1)}\left(\frac{1}{\ln \frac{\kappa}{\left|X_{0}-Y_{0}\right|}}-\frac{\int_{0}^{t} a(s) \mathrm{d} s}{\left(\int_{0}^{t}|b(s)| \mathrm{d} s\right)^{p}}\right)^{t}} . \\
=\frac{\left.\left(\int_{0}^{t}|b(s)| \mathrm{d} s\right)^{p}\right)^{2}}{\left(\int_{0}\right.}
\end{gathered}
$$


Since

$$
\limsup _{T \rightarrow+\infty} \frac{\int_{0}^{t} a(s) \mathrm{d} s}{\left(\int_{0}^{t}|b(s)| \mathrm{d} s\right)^{p}}<0 \text { and } \int_{0}^{+\infty}|b(s)| \mathrm{d} s=+\infty
$$

we get

$$
\begin{aligned}
\lim _{t \rightarrow+\infty} & M\left\{\left|X_{t}-Y_{t}\right|>\kappa\right\} \\
= & \lim _{t \rightarrow+\infty} \frac{1}{\left(\int_{0}^{t}|b(s)| \mathrm{d} s\right)^{2(p-1)}\left(\frac{\ln \frac{\kappa}{\left|X_{0}-Y_{0}\right|}}{\left(\int_{0}^{t}|b(s)| \mathrm{d} s\right)^{p}}-\frac{\int_{0}^{t} a(s) \mathrm{d} s}{\left(\int_{0}^{t}|b(s)| \mathrm{d} s\right)^{p}}\right)^{2}}=0 .
\end{aligned}
$$

Therefore for any given $\kappa$ and $\varepsilon$, there exists a number $N$ such that $M\left\{\left|X_{t}-Y_{t}\right|>\kappa\right\} \leq \varepsilon$ when $t>N$. When $0<t \leq N$, there exists a number $\delta$, say

$$
\delta=\kappa \exp \left(-\frac{\sqrt{3}}{\pi} \int_{0}^{+T}|b(s)| \mathrm{d} s \ln \frac{1-\varepsilon}{\varepsilon}+\sup _{0 \leq t \leq N} \int_{0}^{t} a(s) \mathrm{d} s\right)
$$

such that

$$
\begin{aligned}
M\left\{\left|X_{t}-Y_{t}\right|\right. & >\kappa\}=M\left\{\left|X_{0}-Y_{0}\right| \exp \left(\int_{0}^{t} a(s) \mathrm{d} s+\int_{0}^{t} b(s) \mathrm{d} C_{s}\right)>\kappa\right\} \\
& =M\left\{\int_{0}^{t} b(s) \mathrm{d} C_{s}>\ln \frac{\kappa}{\left|X_{0}-Y_{0}\right|}-\int_{0}^{t} a(s) \mathrm{d} s\right\} \\
& =1-\Phi_{t}\left(\ln \frac{\kappa}{\left|X_{0}-Y_{0}\right|}-\int_{0}^{t} a(s) \mathrm{d} s\right) \\
& =1-1-\Phi_{t}\left(\ln \frac{\kappa}{\delta}-\int_{0}^{t} a(s) \mathrm{d} s\right) \\
& \leq \varepsilon
\end{aligned}
$$

as $\left|X_{0}-Y_{0}\right|<\delta$. Thus the uncertain differential Eq. (6) is stable. Furthermore, it is obvious that

$$
\begin{gathered}
\limsup _{T \rightarrow+\infty} \frac{\int_{0}^{t} a(s) \mathrm{d} s+\int_{0}^{t} b(s) \mathrm{d} C_{s}}{\left(\int_{0}^{t}|b(s)| \mathrm{d} s\right)^{p}} \leq \lim _{T \rightarrow+\infty} \sup _{t \geq T} \frac{\int_{0}^{t} a(s) \mathrm{d} s}{\left(\int_{0}^{t}|b(s)| \mathrm{d} s\right)^{p}} \\
+\limsup _{T \rightarrow+\infty} \frac{\int_{0}^{t} b(s) \mathrm{d} C_{s}}{\left(\int_{0}^{t}|b(s)| \mathrm{d} s\right)^{p}}<0, \text { a.s. }
\end{gathered}
$$

Since $\int_{0}^{+\infty}|b(s)| \mathrm{d} s=+\infty$, we have $\lim _{t \rightarrow+\infty} \int_{0}^{t} a(s) \mathrm{d} s+\int_{0}^{t} b(s) \mathrm{d}$ $C_{s}=-\infty$, a.s. Thus

$$
\begin{aligned}
\lim _{t \rightarrow+\infty}\left|X_{t}-Y_{t}\right| & =\lim _{t \rightarrow+\infty}\left|X_{0}-Y_{0}\right| \exp \left(\int_{0}^{t} a(s) \mathrm{d} s+\int_{0}^{t} b(s) \mathrm{d} C_{s}\right) \\
& =\left|X_{0}-Y_{0}\right| \lim _{t \rightarrow+\infty} \exp \left(\int_{0}^{t} a(s) \mathrm{d} s+\int_{0}^{t} b(s) \mathrm{d} C_{s}\right) \\
& =0 \text { (a.s.) }
\end{aligned}
$$

Therefore uncertain differential Eq. (6) is asymptotically stable.

Example 3. Suppose that $a(t)$ and $b(t)$ are two functions defined by

$$
a(t)=-t^{p-1}, p>1 \text { and } b(t)=1 .
$$

Then

$$
\limsup _{T \rightarrow+\infty} \frac{\int_{0}^{t} a(s) \mathrm{d} s}{\left(\int_{0}^{t}|b(s)| \mathrm{d} s\right)^{p}}=-1 \text { and } \int_{0}^{+\infty}|b(s)| \mathrm{d} s=+\infty .
$$

Thus the uncertain differential equation

$$
\mathrm{d} X_{t}=-t^{p-1} X_{t} \mathrm{~d} t+X_{t} \mathrm{~d} C_{t}
$$

is asymptotically stable.

Theorem 6. Let $a(t)$ and $b(t)$ be two continuous functions. Then the uncertain differential equation

$$
\mathrm{d} X_{t}=a(t) X_{t} \mathrm{~d} t+b(t) \mathrm{d} C_{t}
$$

is stable if $\int_{0}^{t} a(s) \mathrm{d} s>-\infty$.

Proof. The uncertain differential Eq. (9) has a unique solution

$$
X_{t}=\exp \left(\int_{0}^{t} a(s) \mathrm{d} s\right)\left(X_{0}+\int_{0}^{t} b(s) \exp \left(-\int_{0}^{s} a(r) \mathrm{d} r\right) \mathrm{d} C_{s}\right)
$$

which is a normal uncertain variable with expected value 0 and variance

$$
\left(\exp \left(\int_{0}^{t} a(s) \mathrm{d} s\right) \int_{0}^{t}|b(s)| \exp \left(-\int_{0}^{t} a(r) \mathrm{d} r\right) \mathrm{d} s\right)^{2} .
$$

Since

$$
\sup _{t \geq 0}\left|\int_{0}^{t} a(s) \mathrm{d} s\right|<+\infty \text { and } \int_{0}^{+\infty}|b(s)| \mathrm{d} s<+\infty
$$

we have

$$
\exp \left(\int_{0}^{t} a(s) \mathrm{d} s\right) \int_{0}^{+\infty}|b(s)| \exp \left(-\int_{0}^{t} a(r) \mathrm{d} r\right) \mathrm{d} s<+\infty .
$$

For any positive numbers $\kappa$ and $\varepsilon$, there exists a number $\delta$, say

$$
\delta=\varepsilon
$$

such that 


$$
\begin{aligned}
& M\left\{\left|X_{t}-Y_{t}\right|>\kappa\right\}=M\left\{| \operatorname { e x p } ( X _ { 0 } ) - \operatorname { e x p } ( Y _ { 0 } ) | \operatorname { e x p } \left(\int_{0}^{t} a(s) \mathrm{d} s\right.\right. \\
& \left.\left.+\int_{0}^{t} b(s) \exp \left(-\int_{0}^{s} a(r) \mathrm{d} r\right) \mathrm{d} C_{s}\right)>\kappa\right\} \\
& =M\left\{\int_{0}^{t} b(s) \exp \left(-\int_{0}^{s} a(r) \mathrm{d} r\right) \mathrm{d} C_{s}>\ln \frac{\kappa}{\left|\exp \left(X_{0}\right)-\exp \left(Y_{0}\right)\right|}-\int_{0}^{t} a(s) \mathrm{d} s\right\} \\
& =1-\Phi_{t}\left(\ln \frac{\kappa}{\left|\exp \left(X_{0}\right)-\exp \left(Y_{0}\right)\right|}-\int_{0}^{t} a(s) \mathrm{d} s\right) \\
& \leq 1-\Phi_{t}\left(\ln \frac{\kappa}{\delta}-\int_{0}^{t} a(s) \mathrm{d} s\right) \\
& \leq \varepsilon
\end{aligned}
$$

as $\left|X_{0}-Y_{0}\right|<\delta$. Thus the uncertain differential Eq. (9) is stable.

Example 4. Suppose that $a(t)$ and $b(t)$ are two functions defined by

$$
a(t)=\frac{1}{\left(t+a_{0}\right) \ln ^{2}\left(t+a_{0}\right)}, a_{0}>0 \text { and } b(t)=\frac{1}{1+t^{2}} .
$$

Then

$$
\left|\sup _{t \geq 0} \int_{0}^{t} a(s) \mathrm{d} s\right|<+\infty \text { and } \int_{0}^{+\infty}|b(s)| \mathrm{d} s=\frac{\pi}{2} .
$$

Thus the uncertain differential equation

$$
\mathrm{d} X_{t}=\frac{X_{t}}{\left(t+a_{0}\right) \ln ^{2}\left(t+a_{0}\right)} \mathrm{d} t+\frac{1}{1+t^{2}} \mathrm{~d} C_{t}
$$

is stable.

\section{CONCLUSION}

The concepts of stability and asymptotical stability for uncertain differential equation were introduced in this paper. Based on them, thestability theorems for linear uncertain differential equation were studied. Fortunately, several sufficient and necessary conditions of stability for linear uncertain differential equations were obtained.

\section{ACKNOWLEDGMENTS}

This work was supported by National Natural Science Foundation of China (No. 61074193) and Nankai University Project Funds for Young Teachers (No. NKQ 1118).

\section{REFERENCES}

Chen, X. and Liu, B. (2010), Existence and uniqueness theorem for uncertain differential equations, Fuzzy Optimization and Decision Making, 9(1), 69-81.

Chen, X. (2011), American option pricing formula for uncertain financial market, International Journal of Operations Research, 8(2), 32-37.

Gao, Y. (2012), Existence and uniqueness theorem on uncertain differential equations with local lipschitz condition, Journal of Uncertain Systems, 6(3), 223232.

Ito, K. (1951), On stochastic differential equations, $\mathrm{Me}$ moirs of the American Mathematical Society, 4, 151.

Kushner, H. J. (1967), Stochastic Stability and Control, Academic Press; New York, NY.

Kats, I. and Krasovskii, N. (1960), On the stability of systems with random parameters, Journal of Applied Mathematics and Mechanics, 24(5), 12251246.

Khas'minskii, R. Z. (1962), On the stability of the trajectory of markov processes, Journal of Applied Mathematics and Mechanics, 26(6), 1554-1565.

Khas'minskii, R. Z. (1980), Stochastic Stability of Differential Equations, Kluwer Academic Publishers, Dordrecht.

Kunita, H. and Watanabe, S. (1967), On square integrable martingales, Nagoya Mathematical Journal, 30, 209-245.

Liu, B. (2007), Uncertainty Theory (2nd ed.), SpringerVerlag, Berlin.

Liu, B. (2008), Fuzzy process, hybrid process and uncertain process, Journal of Uncertain Systems, 2(1), 3-16.

Liu, B. (2009), Some research problems in uncertainty theory, Journal of Uncertain Systems, 3(1), 3-10.

Liu, B. (2010), Uncertainty Theory: A Branch of Mathematics for Modeling Human Uncertainty, SpringerVerlag, Berlin.

Liu, Y. (2012), An analytic method for solving uncertain differential equations, Journal of Uncertain Systems, 6(4), 244-249.

Meyer, P. A. (1970), Séminaire de ProbabilitésIV Université de Strasbourg, Springer-Verlin, Heidelberg.

Peng, J. and Yao, K. (2011), A new option pricing model for stocks in uncertainty markets, International Journal of Operations Research, 8(2), 18-26.

Zhu, Y. (2010), Uncertain optimal control with application to a portfolio selection model, Cybernetics and Systems, 41(7), 535-547. 